\title{
Some Aspects of the Protozoa and their Way of Life The Sixth Marjory Stephenson Memorial Lecture
}

\author{
By MURIEL ROBERTSON \\ Institute of Animal Physiology, Babraham Hall, Babraham, Cambridge \\ INTRODUCTION
}

I feel much honoured to have been invited to give the Marjory Stephenson Memorial lecture of 1963, but I must confess that I also approach the undertaking with considerable trepidation as it fills me with an acute sense of inadequacy.

In 1961 Professor Knight in his lecture on the growth of microbiology gave a very good account of Marjory Stephenson's own contribution and her influence on the development of the subject. She had a wide and catholic view of the study she had so much at heart. Microbiology, in her view, was to embrace the most various aspects of the investigation into the way of life found in micro-organisms.

I feel therefore that I may be allowed today to direct your attention to a large group of extremely diverse organisms collected under the general title of Protozoa.

This large phylum can be considered as made up of micro-organisms. Their size varies from minute creatures only a few microns in length to relatively large organisms, such as some of the ciliates, visible to the naked eye. It is rather futile to debate the question as to whether they are cells or not. They have, so to speak, the furniture of cells. The point, however, is that they are living creatures which carry out their complicated or relatively simple lives, as the case may be, in sequence. By that I mean that the stages follow each other in general in time, rather than being bound up in one organism the identity of which is laid down from the start. A protozoon divides and there are two but no parent remains; the parent does not die: it just disappears. A microgamete fuses with a macrogamete to form a zygote which ultimately divides up into a number of spores which carry on the cycle, but the zygote has disappeared. The span of life of the protozoa therefore is a debatable matter. Apart from violent death, it is either the period between divisions, or other forms of reproduction, or it is co-existent with the life of the planet from the time of the emergence of the organism.

The protozoa have been conditioned by the fact that they require a fluid or very moist environment. This need has influenced their nature and also limited their development. A great many protozoa produce cysts which are very resistant and can survive a considerable degree of desiccation; the degree of resistance, however, differs very much in different species. The protozoa have developed in the most versatile way and have, as parasites, penetrated into a variety of body fluids and tissues and also into unexpected environments. The parasitic Cnidospora, for example, live in the gall bladder of fishes and tortoises, and certain free-living amoebae were found by Dr Hindle in the hot springs at Dax in Southern France, at a temperature of $54^{\circ}$ (Hindle, 1932). Dr Hindle collected these and made cultures of them; one of which he kindly gave to me. In my sample there was a small lively amoeba of the Hartmanella type. The technique for survival of the one I had was 
characterized by a very remarkable readiness to encyst, showing that the range of conditions for active life was narrow.

Out of the immense variety of the biological capacity shown by the protozoa I propose to select a few examples of the ingenuity of their relation to their environment, and their pioneering spirit in the evolution of basic methods, which suggest patterns for the biological development found in many higher organisms.

The examples I propose to consider are:

(1) The character of the protozoan cell, the function of the nucleus and some of the varieties of nuclear division.

(2) The highly experimental approach of the phylum to sex.

(3) A consideration of some of their life-cycles and the capacity to rationalize certain of the complicated procedures, when a simpler method would appear to serve the purpose of survival.

It is obvious that these matters can only be treated rather schematically in the space at my disposal.

(1) The protozoan cell presents itself under a very large number of forms, but for the moment I want to consider the free-living less complicated types. Inevitably the prototype that comes to hand is one of the larger amoebae such as Amoeba proteus. The organism as seen under the light microscope is a gelatinous mass with various inclusions. It is contained within a double membrane called the plasmalemma which is only visible in an electron micrograph. The ground substance of protoplasm and its submicroscopic make-up is of such a complexity that no brief analysis is adequate; it is beyond the scope of this lecture and the competence of the lecturer. But it would seem to be responsible for the gel and sol activity of, for example, the pseudopodia and also for the internal movements so frequently observed in the protoplasm (Frey-Wyssling, 1948, 1955; Grell, 1956; Kopac, 1950; Landau, Zimmerman \& Marland, 1954).

The peripheral membrane of the amoeba is under some interesting and obscure control and I have failed to find any explanation of the well-known fact that one can tell when an amoeba of this type is about to divide by observing that instead of rolling and lolling about it pulls itself together into a neat mulberry shape with prominent knobs. Moreover, it does this nearly always at night.

In the last decade Brandt (1958) and Schumaker (1958) have carried out studies on Amoeba. Brandt calls the form he worked with Amoeba chaos chaos, but in this country it passes by the less spectacular name of $A$. proteus, which is used by Schumaker. Brandt showed that the amoeba has a capacity to absorb protein through the plasmalemma or surface membrane. This occurs by the formation of tunnels into the cytoplasm by the invagination of the membrane. These tunnels become cut off and are broken up into droplets which are absorbed in the protoplasm. The process is known as pinocytosis. It was found to be induced by charged molecules which included many proteins and salts. In Schumaker's 1958 study the uptake, of two proteins, ribonuclease and cytochrome, was observed in great detail. His explanation of the results involved the binding of the protein molecules to the external membrane by a process which he considered to be pinocytosis. It would seem that these studies might form a step towards the understanding of the physiology of the large number of organisms which the protozoologists happily describe as saprophytic. These do not ingest food in an obvious 
way and are contrasted with the holozoic organisms whose food is taken in by straightforward, often visible, methods and digested in food vacuoles (Steinert \& Novikoff, 1960).

The protozoan cell, in addition to the nucleus, contains a number of formed elements and structures varying somewhat with the species, such as microsomes, the Golgi apparatus and various types of organelles. Those found most frequently in the protozoa comprise cilia, flagella, mitochondria, cytoplasmic membranes and plastids; these are essentially, as recently emphasized by Grimstone, similar in their fine structure to those of higher plants and animals (Grimstone, 1961). In spite of the importance of all the organelles, the general functioning of the cell is ultimately dependent on the nucleus, which contains the chromosome complex and the all important DNA. While this is in general true, researches by Brachet and his co-workers have shown that it is not immediately essential for the continued life of the cell. And it does not seem to be true that the nucleus is the only source of protein synthesis. He showed that in an amoeba divided so as to produce a nonnucleated and a nucleated half the oxygen consumption of the two fragments remained alike for at least 10 days. In the case of a unicellular alga Acetabularia mediterranea growth and protein synthesis proceeded at the same rate in both the nucleated and the non-nucleated halves during 2-3 weeks (Brachet, 1954, 1957).

In general the protozoan cell has one nucleus, but occasionally there may be more. These can be of the same structure and type as in the big amoeba Pelomyxa, to give only one example, which has a large number of similar nuclei. There can also, as in the ciliates, be a small micronucleus concerned with the heritable characters and active in nuclear recombination at conjugation, and the large trophic nucleus. This last is of a type with a different make-up from that found in the other groups of the protozoa and must be considered later.

The structure of the nucleus as shown in the light microscope in both living and stained preparations shows a considerable variety and they cannot all be gone into in detail. But in general there are two main types. One is the reticulate nucleus, in which the chromatin appears as granules or threads lying within the nuclear membrane often without a visible nucleolus. The other is the very frequent vesicular nucleus with a large karyosome now more usually called the nucleolus.

The chromatin, from which the chromosomes later appear in the more developed forms, is often, in the more primitive organisms, apparently diffused throughout the nuclear sap in the form of small particles which can be recognized by the Feulgen reaction. The chromosomes vary in the protozoa from well-defined structures comparable with those found in the metazoa to ill-defined aggregations of chromatin granules.

In this rapid review I propose only to describe the division of two small flagellates, both of which I have had the opportunity of investigating myself. In the resting state they both have typical vesicular nuclei but their mitotic figures are widely different. They are Bodo caudatus and Heteromita globosa (Robertson, 1927, 1928).

Bodo is the more primitive type and the chromatin is never visible in the form of recognizable chromosomes. It is a biflagellate with unequal flagella, each as usual with a basal granule. In addition, there is a kinetoplast which is Feulgen positive.

In the non-dividing nucleus there is a large dense nucleolus which does not stain in the Feulgen reaction and an arrangement of Feulgen-positive granules lying 
between the nucleolus and the nuclear membranes. At division the single large nucleolus does not break up but keeps its identity throughout the process. In the early stage the nucleolus comes to lie at one side of the nuclear space and the chromatin, which now stains with much greater intensity, is aggregated on the opposite side, still within the nuclear space.

The nucleolus gradually moves into the chromatin mass, which gets pushed apart into two segments which come to lie at either end of the solid elongated bar of the nucleolus. This now elongates further, producing a dumb-bell figure, becoming increasingly thin at the centre, while the chromatin now lies as two separate masses at either end of the drawn-out nucleolus. The two nuclei finally draw apart and are now reconstructed in the original form. The kinetoplast has by now also divided and the body of the flagellate divides. The important point is that the nucleolus never disappears in this division, there is no equatorial plate nor are visible chromosomes or separate chromatin granules formed. In Heteromita globosa, which is also a biflagellate with two unequal flagella, the resting nucleus has again a large single nucleolus and the chromatin is arranged, as in Bodo, between the cell membrane and the nucleolus. At division, however, the nucleolus disappears and there is produced a perfect mitotic spindle, at the equator of which there are assembled small lumps of chromatin on an orthodox equatorial plate. At the apices of this figure the basal granules of the two flagella are found to have moved apart and are now functioning as centrosomes, one showing the shorter of the two unequal flagella and the other the longer one. The chromosomes, as we now call the Feulgen-positive little masses at the equator of the spindle, reduplicate and move apart to either pole, the nuclei are reconstructed in the usual way and the organism divides. The nucleolus reappears again as a large single structure in the centre of the nucleus. The centrosome-basal-granules now reduplicate themselves and the appropriate second flagellum grows out. In passing I should like to point out the double function of the basal granules of the flagella, both as basal granules of flagella and as centrosomes. This also occurs in the flagellated collar cells of certain sponges (Robertson, 1911a).

The point I wish to make concerning these two small flagellates is that one provides an example of a primitive form of nuclear division with a persisting nucleolus and the other a perfect type of mitosis with a disappearing nucleolus, which in all the broad essentials conforms to that found in higher types of organisms.

While I am considering the behaviour of some of the protozoan nuclei, I should like to mention some experiments carried out by Cleveland (1956).

He showed that in Trichonympha (one of the Polymastigote flagellates) the chromosomes could be completely destroyed by exposure to an appropriate concentration of oxygen (70-80\%) during the early period of gametogenesis when the chromosomes were reproducing themselves. This treatment does not damage the cytoplasm nor its organelles. The organism will live from three to five days and is capable of carrying on the differentiation towards gametogenesis as regards all the activities such as encystation, loss and renewal of the parabasals and flagella. The centrosomes or centrioles, as Cleveland prefers to call them, moreover function in a normal manner in the production of an achromatic spindle. Finally the cytoplasm can be effectively divided to produce two anucleate gametes; these of course 
eventually die. It should be explained that in this species two gametes, a male and a female, which later escape, are formed within the one cyst.

These experiments throw an interesting light on the autonomy of the organelles and the achromatic structure from the immediate influence of the chromosomes and the DNA which they contain. I think the word 'immediate' must not be forgotten in considering these results.

(2) Sex in the protozoa. What we have to consider at this stage is not so much sex as what underlies it, namely the importance of biparental inheritance. As we find the protozoa today many species, both free living and parasitic, do not show any sign of it. What is meant by biparental inheritance is that at some stage in their life cycle there is a fusion of two reduced nuclei to make a new single one, thus obtaining the genetic material from two different sources.

Biparental inheritance, with the range of variation it implies in the possible recombination of the genetic material, has obvious potentialities in the creation of variants. It can also probably operate as a stabilizing agent for the species by the elimination of nuclear material at the reduction division.

The protozoa in which one finds biparental inheritance approach the matter in a number of very different ways. Some of the flagellates for example are haploid, that is to say they have only half the number of chromosomes all their lives except for a brief period during which they come together to form the zygote. Reduction to the haploid state occurs at the first nuclear division after syngamy. In these flagellates there is no observable difference between the two gametes and they are designated by the symbols + and - . There is, however, an important physiological distinction and clones made from one of the products of the first division of the zygote are unable to mate and form zygotes within the clone. It is only when gametes from a + clone meet gametes from a - clone that syngamy occurs and zygotes are formed.

One of the very simplest forms of syngamy occurs when as with Polytoma uvella the two haploid flagellates come together and, in a matter of minutes, both throw off their flagella and join by their anterior ends and fuse into a round shape and encyst, the two nuclei fusing as two small balls. The process can be observed over and over again by adding a little distilled water to a well-fed culture in decomposing hay and putting a few $\mathrm{ml}$. into an open watch-glass. The syngamy and cyst formation gets going in about $10 \mathrm{~min}$. The environment was changed, I may say in passing, by adding the distilled water and by the exposure to the air in the watchglass. No doubt also the Polytoma were carrying some form of distinction, probably of the + and - kind.

We have been dealing with organisms that have a definite, almost a pedantic, outlook on biparental inheritance.

In Actinophrys, however, which is one of the beautiful freshwater heliozoa, there is a curious form of syngamy called paedogamy. This organism throws over the biparental idea altogether and carries on the process within the one cell. Berlarr made a long and very excellent study of this organism in 1923 and 1924. Under certain conditions which he could control a single individual produces a cyst within which the organism, which is diploid, undergoes division into two; the two halves lying within the one cyst undergo two nuclear divisions of which the first is the reduction division whereby the diploid number of chromosomes is reduced to half. 
In these nuclear divisions, as is usual, the unwanted halves degenerate and disappear. There is an interesting point raised by this disappearance of the surplus nuclei. It occurs at this stage in very many protozoan life cycles. The redundant nuclei are quickly digested in the protoplasm, but what difference is there in the definitive surviving nucleus in each gamete that prevents it sharing the fate of its fellows? The two now uninucleate organisms come together, the protoplasms fuse and the two nuclei unite into one diploid syncarion. Belarr found that one of the gametes after maturation was more lively than the other and he considered it to be the male gamete.

The German protozoologists of that period were much exercised by the bisexuality of the nuclear material. But it was never a very clear conception and I do not feel that it was very well established. The Actinophrys zygote secretes a protective cyst which upon being removed to fluid of lesser osmotic pressure germinates and the Actinophrys proceeds upon its active way again (Bělăr, 1923, 1924).

Belăr's study is of particular value because he kept the organism under observation for a long time and he got a real insight into the way of life of this free-living Heliozoon. He found that syngamy could be induced or prevented according to the adjustment of the conditions of culture, and also that under uniformly favourable conditions Actinophrys could be cultivated indefinitely without syngamy or any other form of nuclear reorganization, beyond that of normal mitotic division. He actually kept a culture for 2 years and 8 months dividing normally, during which time there were 1244 generations without syngamy, and then he induced it again by the alteration of the external conditions. Here we have an organism with a definite if somewhat unusual type of syngamy, the expression of which is dependent upon the external stimulus of the environment, and which could be suppressed for an indefinite period without damage to the organism if the surrounding conditions were stabilized and kept uniformly favourable. This dependence on change in the environment emphasizes the point raised earlier that the protozoa carry out their lives in sequence in relation to the environment. Hartmann, in 1928, brought this aspect of the way of life of the protozoa to its logical conclusion and the life-cycle to the vanishing point, in his study of Amoeba proteus (Hartman, 1928). He maintained a single amoeba in a condition of active life without even permitting it to divide by cutting off a portion of the protoplasm every day. The regeneration of the protoplasm kept the cell in equilibrium for 130 days without division, when he gave up the experiment. Parallel experiments showed that these amoebae, if left undisturbed or if an insufficient amount of protoplasm had been eliminated, divided quite normally even after many days of this inhibited type of culture.

In tracing the emergence of the gamete types and the development of sex there is a great deal of material to choose from.

The method of association of two mature organisms in one reproductive cyst is found in certain parasitic forms, notably among the large group of the parasitic Gregarines, which are a subgroup of the Sporozoa. This is quite different from the paedogamy which we have seen in Actinophrys. In, for example, Lipocystis polyspora, parasitic in the fly Panorpa communis, after a phase of schizogony or asexual division, larger but apparently similar organisms are formed which are the gametocytes. They come together in pairs which form a single cyst but do not fuse into a single organism. In each organism the nucleus divides repeatedly, but the proto- 
plasm does not. In each of the multinuclear gametocytes there now occurs a process whereby the nuclei bud off or are segregated into individuals consisting of one nucleus surrounded by a small portion of protoplasm. How this actually happens is one of the darker mysteries of protozoan physiology. The gametes from each of the gametocytes are now free in the common cyst; they fuse in pairs, one from each of the two gametocytes, and finally develop into sporozoites each containing 8 spores. Here, where the syngamy is perfectly clear, the differentiation of the sexes is nevertheless not obviously manifest, as the distinction between the final gametes is not well developed (Grell, 1938).

There are a number of interesting stages in the Coccidia, which are also a suborder of the Sporozoa, which show the development of the differences between the microgametes and the macrogametes, but I cannot go into all of them now.

Eimeria schubergi, however, parasitic in a centipede, for example, shows the final stage in the differentiation in the gametes. Here the macrogametocyte develops into a large macrogamete with finally one mature haploid nucleus. The microgametocyte produces a relatively large number of highly motile microgametes, one of which penetrates into the large macrogamete and contributes only the male nucleus, which becomes associated with the female nucleus on the fertilization spindle. The fertilized zygote now develops into the ookinete, in which four spores are developed, each with two sporozoites. The ripe sporocyst develops a very resistant cyst, which is evacuated to the exterior in the hope that it will be ingested by another centipede (Schaudinn, 1900).

The malarial parasite, also belonging to a subclass of the Sporozoa, has a lifecycle which in its broad outline resembles that of the Coccidia. It is more complicated, but also culminates in a macrogamete which is penetrated by a motile microgamete which delivers the male nucleus. This occurs in the gut of the mosquito which acts as the vector in transmitting the parasite.

The electron microscope studies by Professor Garnham and his co-workers of the sporozoites which are formed in the ookinete, and which are finally delivered from the salivary gland of the mosquito into the vertebrate host, are revealing a very complicated morphological make-up.

This is not part of my theme, but I should like to point out the latent morphogenetic capacity of the nuclear material which awaits the appropriate stimulus to come into action (Garnham, Bird \& Baker, 1960; Garnham, Bird, Baker \& Bray, 1961; Garnham, Bird \& Baker, 1962).

To return to the type of syngamy exhibited by these organisms. We have here the full development of a female egg cell or macrogamete, which is responsible for the building material of the coming generation as well as contributing the female nuclear element, and a well-developed motile microgamete which plays a kinetic and purely nuclear role.

This again shows the pattern later found in so many of the higher forms, but I do not wish it to be considered that any of these arrivals at a mechanism found to be general in the metazoa has of necessity any philogenetic significance. My point is that the mechanism is arrived at by the protozoa and has also proved the appropriate one for the more developed organisms.

This matter has been well treated recently by Grimstone (1959), who sums up as follows: 'It is suggested that at the lower levels of organization exemplified by 
biochemical characters, cell organelles, and early embryonic forms, similarities do not necessarily imply common ancestry, independent origin is equally probable'.

While we are dealing with sex there is one great class of the protozoa, namely the ciliates, which have arranged their nuclear outfit by dividing the trophic and sexual functions. Thus there are two nuclei in all the ciliates; the micronucleus, which in many types is very small, carries the genetic material which is responsible for hereditary characters, and the large macronucleus, which is responsible for the metabolism and trophic functions in general. The ciliates, instead of developing into male and female gametes which fuse as separate organisms to form a zygote, keep their identity and exchange a small nucleus which has been reduced to the haploid state by the usual process of maturation. The general arrangement of conjugation is familiar and need not be repeated here. The important point is that when the conjugating pair separate the exconjugates have each its old body, but a reconstructed new nucleus made up by the fusion of its own reduced nucleus with the wandering nucleus of the partner. The old macronucleus has been digested by this time. After some divisions (usually two) of the syncarion the definitive micronucleus is produced and in addition one of the micronuclei develops into the macronucleus by a process of chromosome reduplication without any spindle formation; thus producing a polyploid nucleus which may be of very large dimensions. The chromosomes in the formation of the macronucleus become aggregated into small bundles called genomes, each of which contains all the genetic material of the original nucleus. How the genomes are held together is not known (Piekarski, 1939, 1941 ; Grell, 1949, 1952, 1953; Fauré-Fremiet, Rouiller \& Gauchery, 1957).

In this kind of polyenergid nucleus endomitosis takes place whereby the individual genomes divide without any spindle. This arrangement permits, first, the growth of the macronucleus, which in some of the larger species can be very large indeed; and, then, for its division without any elaborate mechanism. At cell division the mass shows a narrowing at the middle and in, for example, Tetrahymena pyriformis it elongates and divides, the two ends draw apart while the central junction is often left in the protoplasm, where it is digested. The development of the macronucleus and its fine structure is being actively studied at the present time. The work is of great theoretical interest, but it is not in a state as yet to be brought together in a survey of this kind.

With the curious passion for simplification which one encounters quite frequently in the protozoa, a group of ciliates-the Tetrahymena-are found, in both the wild state and particularly in cultivation in bacteria-free media, to dispense with the micronucleus. These strains never conjugate but they do not degenerate. The two species Tetrahymena vorax and $T$. patula L-FF, of which I have two amicronucleate strains, are dimorphic and also produce transparent (indeed they are rightly called invisible) cysts from which emerge eight small creatures known as tomites (Corliss, 1953; Williams, 1960, 1961). They manage all this and maintain their character without the sexual nucleus. The geneticists consider these amicronucleate types to be senescent forms, but they live happily for years and as I have said are frequently found wild in nature (Elliott, Addison \& Cary, 1962). I have myself kept the monomorphic $T$. pyriformis strain GLR for a matter of 28 years and the two dimorphic forms each for 12 years.

The ciliates are definitely a tribe apart; they are extremely numerous and have an 
extraordinary range of morphological types. Their development of cilia and organelles and their power of contraction are all remarkable. They are mostly free living, many of them are terrible predators and cannibals and in addition there are also a good many successful parasitic types.

(3) Life-cycles. I would like now to mention the life-cycles of some of the Trypanosomes. The appearance of the Trypanosome, as seen with the light microscope, is well known. The Trypanosomes belong to the group of the Trypanosomids, which is made up of four species: the Leishmania, which are without a flagellum for at least a good deal of their life; the Leptomonads with the kinetoplast at the anterior end and a free flagellum; the Crithidia with the kinetoplast still anterior or very near to the nucleus and the Trypanosomes proper with the kinetoplast at the posterior end and the flagellum running towards the anterior of the body with a free end.

The Trypanosomes are blood parasites and have a very wide distribution. They are present in fishes, in frogs and lizards and crocodiles and birds; in mammals from mice to elephants and in monkeys and man. There are, however, a few strange omissions. They have, so far as I am aware, not been found in newts in England and Europe, but are found in newts in America, where curiously enough they have not been found in fishes, at least in freshwater fish. This is probably due to idiosyncrasies in the vectors available for transmission (Barrow, 1953).

Trypanosomes were probably derived from leptomonad and crithidial forms inhabiting plant-sucking insects and various invertebrates. There is an interesting form called Strigomonas oncopelti, a leptomonad which is found in an African plantsucking bug and in the latex channels of the plant on which the insect feeds. I mention it here because it has been a suitable subject for biochemical studies by Dr Newton.

The life-cycles of the Trypanosomes vary according to the nature of the vertebrate host and the type of transmitting agent, but the vast majority are transmitted by some blood-sucking vector and, before they are viable and, as it were, ripe for transmission to another host, they undergo a morphological change. They pass through a crithidial stage in which the kinetoplast has come to lie much nearer the anterior end of the organism. And upon regaining the trypanosome state they are once more ready to be delivered to the blood stream of the vertebrate host.

In, for example, the leeches which transmit the trypanosomes of tortoises and fishes, the whole story takes place in the intestine and the crop. The flagellates taken in with the blood go through a crithidial stage; some indeed become rounded off and go through a period of quiescence, but develop through a leptomonad phase and again into a crithidial state. All this group finally again assume the trypanosome shape, become very slender and elongated and pass from the crop into the salivary fluid in the proboscis and are injected into the fish (Robertson, 1911 $b$ ).

In the Gambiense-Brucei group, the trypanosomes divide and multiply in the intestine of the tsetse fly, and, while they undergo a slight alteration in shape, do not go through the crithidial phase until they migrate forward into the proboscis and then into the salivary gland. Here, after a period of rapid multiplication as crithidia mostly attached to the gland wall by the flagellum, they turn into small freeswimming trypanosomes which are viable in the blood of the vertebrate (Robertson, 1913). 
It should be mentioned here that the trypanosomes in the fish and the tortoises develop in almost $100 \%$ of the leech intermediate host. Whereas the BruceiGambiense group complete the tsetse fly stage in at most $10 \%$ of the flies and often in only $3 \%$. The tsetse fly, however, remains infective for the duration of its life.

At this point I must digress to remark that the electron microscope has revealed that the elegant simplicity of the trypanosome as seen in the light microscope masks a much more complicated structure. Dr Vickerman has lately shown that the kinetoplast in the blood stream form seems to be connected with a large mitochondrion passing forward towards the anterior end.

In the cultural form, which is thought to correspond, in general opinion, with the midgut stage in the tsetse fly, a second mitochondrion grows out from the kinetoplast towards the posterior end and the authors suggest that this pushes the kinetoplast nearer to the nucleus (Vickerman, 1962; Pittam \& Vickerman, 1962). For the moment the deductions drawn from these morphological changes need further elucidation and it would be better if they were made from the investigation of the organism in the gut of the fly rather than from the cultural forms in vitro.

The cycles of different tsetse-borne African Trypanosomes vary in detail, but are all essentially of the same type, the final fly stage always taking place in the salivary fluid though not always taking place in the salivary gland. The connexion of a kinetoplast with the mitochondria has been recognized for some years, but its function in the cycle in the fly is a new suggestion (Clark \& Wallace, 1960; Steinert, 1960).

But now we come to what can only be called a break out by the three subspecies Trypanosoma evansi, $T$. equinum and $T$. equiperdum of the Brucei group. These trypanosomes have been the subject of a detailed study by Dr Hoare and there is little doubt that this group is derived from the $T$. brucei type. But unlike all the other trypanosomes parasitic in mammals there is no intermediate host cycle. In the case of $T$. evansi the parasites are passed by direct mechanical transference of infected blood from one animal to another on the proboscis of Tabanid flies. $T$. evansi is found in horses, mules, camels, cattle and even in dogs. The subspecies T. equiperdum, which is parasitic in horses, is transmitted by direct contact at coitus. It has caused much loss among horses in Europe, especially in France, and it also spread into Canada, but it has been eradicated from that country about 40 years ago by the very remarkable work of a veterinary officer in 1915 (Watson, 1915). The whole elaborate business of the intermediate host cycle in the tsetse fly has simply been dropped and this triumphant group of trypanosomes has spread over vast areas beyond the distribution of any of the Glossina species. It causes a serious infection in North Africa, the Middle East, Asia, in India and China. In the guise of the subspecies T. equinum it has passed into the New World and is the cause of a disease in horses called Mal de Caderas in Brazil.

The behaviour of Trypanosoma evansi is a striking example of the short circuiting of a life-cycle when a simpler form of transmission meets the essential survival needs of a protozoan species.

Trypanosoma equinum has further simplified things by shedding the kinetoplast while, as is expected, retaining the blepharoplast or basal granule of the flagellum.

The akinoplastic trypanosomes seem to arise originally from individuals in Trypanosoma evansi infections, where at division the kinetoplast fails to divide so 
that one of the products of division has a kinetoplast and the other has not. These last may disappear, being overgrown by the normal type, but in some strains fluctuating numbers of these mutant forms are found and in some cases a strain is found without any of the trypanosomes having a kinetoplast. The strains are perfectly viable and five of these were found in camels in the Sudan in the years 1934 to 1937 (Hoare, 1954, 1956). One of these strains has been maintained for 17 years in passage in mice and has never shown any return to the original condition.

The type of trypanosome known as Trypanosoma equinum, which is found in horses in the Argentine and other parts of South America, was first discovered in 1901. It is indistinguishable from the akinetoplast $T$. evansi strains found in camels in the Sudan. The disease in the New World was first described in Brazil in the middle of the last century derived, it seems, from imported horses. It is transmitted by direct passage by biting flies from animal to animal in the same way as T. evansi.

Occasional mutants without a kinetoplast appear in African Trypanosoma brucei strains, but they disappear and Reichenow has shown that the mutants do not survive in passage by the tsetse fly vector. This would seem to indicate that in the species transmitted cyclically by the tsetse fly these mutants are unable to survive. The loss of the organelle in $T$. equinum with the direct type of transmission has not inhibited the spread of the species, which has been established in South America for about a hundred years.

This akinoplastic condition can be produced artificially by exposing the trypanosomes to acriflavine and also to pyronin, as was first shown by Werbitski in 1910. It is interesting to find that Bodo caudatus, which as already shown is a free-living flagellate with a Feulgen-positive kinetoplast, when exposed to acriflavine produces up to $75 \%$ of akinoplastic organisms (Robertson, 1929); these however do not survive although they can divide once or twice. The drug, which is a bright yellow colour, is taken up by the kinetoplast in vivo and can be observed directly under the light microscope. As the strain becomes fast to the drug the colour ceases to be taken up in vivo. The inability of the Bodos to survive without a kinetoplast may perhaps be related to the presence of a large mitochondrion connected with the kinetoplast in these organisms lately described by Pitelka (1961). This is an electron microscope study and it is not known if all the species of Bodo have this character.

The trypanosomid Strigomonas oncopelti, which lives in the sap of an African latex plant and is transmitted by a plant-sucking bug, was found by Dr Newton to be quite insensitive to acriflavine and the drug is not taken up by the kinetoplast in vivo. Acriflavine will however penetrate into a dead organism staining the whole body (unpublished communication of Dr B. Newton, 1963).

I should like in conclusion to point out in what an interesting state the microbiological investigation of the protozoa is at this moment. The investigator can by means of the electron microscope observe the fine structure of the organism. He can follow the different stages of morphogenesis and in certain instances in time sequences which can be measured in minutes. It is true he does this by the despised methods of observation and description. He has powerful enzymes at his disposal; he can follow the uptake of labelled substances, and he can get some useful evidence from histochemistry. 


\section{REFERENCES}

Barrow, J. H. (1953). The biology of Trypanosoma diemyctyli (Tobey). I. Trypanosoma diemyctyli in the leech Batrachobdella picta (Verrill). Trans. Amer. micr. Soc. 72, 197.

BĔLǍr, K. (1923). Untersuchungen an Actinophrys sol Ehrenberg. I. Die Morphologie des Formwechsels. Arch. Protistenk. 46, 1.

BĚLǍr, K. (1924). Untersuchungen an Actinophrys sol. II. Beiträge zur Physiologie des Formwechsels. Arch. Protistenk. 48, 371.

Brachet, J. (1954). Nuclear control of enzymatic activity. Coulston Symposium of Cell Physiology, p. 91. Bristol: Coulston Soc.

Brachet, J. (1957). Biochemical Cytology. New York: Academic Press Inc.

Brandt, D. W. (1958). A study of the mechanism of pinocytosis. Exp. Cell Res. 15, 300.

Clark, T. B. \& Wallace, F. G. (1960). A comparative study of kinetoplast ultrastructure in the trypanosomatidae. J. Protozool. 7, 115.

Cleveland, L. R. (1956). Cell division without chromatin in Trichonympha and Barbulanympha. J. Protozool. 3, 78.

ConLIss, J. O. (1953). Comparative studies on holotrichous ciliates in the ColpidiumGlaucoma-Leucophrys-Tetrahymena group. Parasitology, 43, 49.

Elurott, A. M., Addison, M. A. \& Cary, S. E. (1962). Distribution of Tetrahymena pyriformis in Europe. J. Protozool. 9, 135.

FaUré-Fremiet, E., Rouiller, C. \& Gauchery, M. (1957). La réorganisation macronucléaire chez les Euplotes. Exp. Cell Res. 12, 135.

Frey-Wyssling, H. (1948). Submicroscopic Morphology of Protoplasma and its Derivatives. Amsterdam and New York: Publ. Comp.

Frey-Wyssling, H. (1955). Die submikroskopische Struktur des Cytoplasma. In Protoplasmatologia. Ed. by L. V. Heilbrunn and F. Weber. Wien: Springer.

Garnham, P. C. C., Bird, R. G. \& Baker, J. R. (1960). I. Electron microscope studies of motile stages of malaria parasites. Trans. R. Soc. trop. Med. Hyg. 54, 294.

Garnham, P. C. C., Bird, R. G., Baker, J. R. \& Bray, R. S. (1961). Electron microscope studies of motile stages of malaria parasites. II. The fine structure of the sporozoite of Laverania (Plasmodium) falcipara. Trans. R. Soc. trop. Med. Hyg. 55, 98.

Garnham, P. C. C., Bird, R. G. \& Baker, J. R. (1962). III. Electron microscope studies of motile stages of malaria parasites. Trans. R. Soc. trop. Med. Hyg. 56, 116.

Grell, K. G. (1938). Untersuchungen an Schizogregarinen. I. Lipocystis polyspora n.g.n.sp., eine neue Schizogragarine aus dem Fettkörper von Panorpa communis. Arch. Protistenk 91, 526.

Grell, K. G. (1949). Die Entwicklung des Macronucleus in Exconjuganten von Ephelota gemnipara R. Hertwig. Biol. Z Zbl. 68, 289.

Grel., K. G. (1952). Der Stand unser Kenntnisse über den Bau der Protistenkerne. (Referat). Zool. Anz. 17, 212.

Grell, K. G. (1953). Die Konjugation von Ephelota gemmipara R. Hertwig. Arch. Protistenk. 98, 287.

Grelt, K. G. (1956). Protozoologie. Berlin: Springer Verlag.

Grimstone, A. V. (1959). Cytology, homology and phylogeny. A note on 'organic design.' Amer. Naturalist, 93, 273.

Grimstone, A. V. (1961). Fine structure and morphogenesis in Protozoa. Biol. Rev. $36,97$.

Hartmann, M. (1928). Utber experimentelle Unsterblichkeit von Protozoen-Individuen. Ersatz der Fortpflanzung von Amoeba proteus. Zool. Jb. 45, 973.

Hindle, E. (1932). Some new thermophilic organisms. J. R. micr. Soc. 52, 133.

HoARE, C. A. (1954). The loss of the kinetoplast by trypanosomes with special reference to Trypanosoma evansi. J. Protozool. 1, 28.

Hoare, C. A. (1956). Morphological and taxonomic studies on mammalian Trypanosomes. VIII. Parasitology, 46, 130.

KNIGHT, B. C. J. G. (1962). The growth of microbiology. J. gen. Microbiol. 27, 357.

Kopac, M. J. (1950). Physical properties of protoplast. Annu. Rev. Physiol. 12, 7. 
Landau, J. V., Zimmerman, A. M. \& Marland, D. A. (1954). Temperature-pressure experiments on Amoeba proteus, plasmagel structure in relation to form and movement. J. Cell. comp. Physiol. 44, 211.

Piekarski, G. (1939). Cytologische Untersuchungen an einem normalen und an einem Micronucleus-losen Stamm von Colpoda steini. Maupas. Arch. Protistenk. 92, 117.

Piekarskr, G. (1941). Endomitose beim Grosskern der Ciliaten. Versuch einer Synthese. Biol. Zbl. 61, 416.

Pitelka, D. R. (1961). Observations on the kinetoplast-mitochondrion and the cytostome of Bodo. Exp. Cell Res. 25, 87.

Pittam, M. D. \& Vickerman, K. (1962). The cultivation of Trypanosoma rhodesiense in liquid medium and the fine structure of the culture forms. Trans. $R$. Soc. trop. Med. Hyg. 56, 270.

RoBertson, M. (1911 a). The division of collar-cells of Calcarea heterocala. Quart. J. micr. Sci. 57, 129.

Robertson, M. (1911b). Transmission of flagellates living in the blood of certain freshwater fishes. Phil. Trans. B, 202, 29.

Robertson, M. (1913). Notes on the life history of Trypanosoma gambiense with a brief reference to the cycles of Trypanosoma manum and Trypanosoma pecorum in Glossina palpalis. Phil. Trans. B, 203, 161.

Robertson, M. (1927). Notes on certain points in the cytology of Trypanosoma raiae and Bodo caudatus. Parasitology, 19, 375.

Robertson, M. (1928). Notes on Heteromita (Protozoa, Flagellata). Parasitology, 20, 10.

Robertson, M. (1929). The action of acriflavine upon Bodo caudatus. Parasitology, 21, 375.

Schaudinn, F. (1900). Untersuchungen über den Generationswechsel bei Coccidien. Zool. Jb. (Abt. 2), 13, 197.

Schumaker, V. N. (1958). Uptake of protein from solution by Amoeba proteus. Exp. Cell Res. 15, 314.

STEINERT, M. (1960). Mitochondria associated with the kinetonucleus of Trypanosoma mega. J. biophys. biochem. Cytol. 8, 542.

Steinert, M. \& Novikoff, A. B. (1960). The existence of a cytostome and the occurrence of pinocytosis in the Trypanosoma mega. J. biophys. biochem. Cytol. 8, 563.

Vickerman, K. (1962). The mechanism of cyclical development in trypanosomes of the Trypanosoma brucei sub-group. An hypothesis based on ultrastructural observations. Trans. R. Soc. trop. Med. Hyg. 56, 487.

Watson, E. A. (1915). Dourine and the complement fixation test. Parasitology, 8, 156.

Werbitski, F. W. (1910). Über blepharoplastlose Trypanosomen. Zbl. Bakt. (Abt. 1), 53, 303.

Williams, N. E. (1960). The polymorphic life history of Tetrahymena patula. J. Protozool. 7, 10.

Wrlliams, N. E. (1961). Polymorphism in Tetrahymena vorax. J. Protozool. 8, 403.

(Delivered before the Society for General Microbiology at its Thirty-seventh Meeting, 8 April 1963) 